\title{
IT CAMP: Abordando Tecnologias da Informação
}

DOI: 10.37702/2175-957X.COBENGE.2021.3517

Fernanda de Jesus Gomes - fernanda.gomes.cdp18310@gmail.com

Centro Federal de Educação Tecnológica de Minas Gerais

Ernesto tognolo 95

31970-170 - BELO HORIZONTE - MG

André Rodrigues da Cruz - andrercruz@gmail.com

Centro Federal de Educação Tecnológica de Minas Gerais

Rua Coronel Agostinho da Fonseca 333

- São Paulo - SP

Sandro Renato Dias - sandrord@cefetmg.br

CEFET MG

Rua Deputado André de Almeida 229

31330-530 - Belo Horizonte - MG

Resumo: Tendo como objetivo atender a comunidade acadêmica e externa, o grupo PET, Programa de Educação Tutorial, da Engenharia de Computação (COMPET) do CEFET - MG situado no Campus Nova Gameleira em Belo Horizonte, procurou introduzir temáticas que envolvem as tecnologias de informação, promovendo um evento, que por meio de palestras, buscou salientar assuntos que envolvem o mercado de trabalho, bem como atualizar os participantes acerca de novas tecnologias. O evento foi nomeado de IT CAMP, com palestras sobre desenvolvimento de jogos; criação de site em WordPress; desenvolvimento de back-end e aprendizado de máquina. Dessa forma, o COMPET findou uma parceria com o Commit Jr, empresa júnior do curso de Engenharia de computação do CEFET-MG, para realizar as atividades do evento que ocorreram entre os dias 24 de Junho e 01 de Julho de 2020, no canal do YouTube do Commit Jr.

Palavras-chave: PET. Engenharia de Computação. Ensino. Extensão. Empresa Júnior. 


\section{IT CAMP: Abordando Tecnologias da Informação}

\section{INTRODUÇÃo}

No cenário de isolamento social, causado pela pandemia do COVID-19, para as universidades, um dos desafios mais valiosos é manter a informação para a comunidade acadêmica. O grupo PET que é sustentado pelo tríade de pesquisa, ensino e extensão, teve como estímulo manter não só a informação, como também seus projetos e atividades.

Nesse contexto, a fim de levar assuntos relevantes e de usabilidade para a universidade, e também para os estudantes externos que se interessam pelo mercado de tecnologia da informação, o COMPET formou uma parceria com o Commit Jr, tendo como objetivo atrair um número maior possível de graduandos no evento, incrementando de forma totalmente online o evento IT CAMP, tendo como principal intuito desenvolver nos participantes conhecimentos e experiências com a área, ou tecnologia, de $\mathrm{TI}$.

Conforme destaca Castells, "As novas tecnologias da informação não são simplesmente ferramentas a serem aplicadas, mas processos a serem desenvolvidos" (CASTELLS, 1999, p. 51). Dessa forma, o projeto IT CAMP foi explanado para toda a comunidade externa, caracterizando-o como projeto de extensão, tendo como intuito desenvolver ainda mais o ensino acerca dos temas abordados, em que os idealizadores do evento puderam contar com o apoio e participação de pessoas que detêm um vasto conhecimento nos assuntos das palestras disponibilizadas.

\section{DESENVOLVIMENTO}

O IT CAMP, realizado entre os dias 24 de Junho a 01 de Julho em 2020, contou com quatro palestras que apresentaram os seguintes temas: desenvolvimento de jogos; criação de site em WordPress; desenvolvimento de back-end e aprendizado de máquina. As palestras foram conduzidas por profissionais da área, docentes do CEFET-MG, e também por representantes da empresa júnior CPE Jr.

\subsection{Atividades desenvolvidas}

\section{Palestra desenvolvimento de jogos}

O mercado de jogos é um dos que mais crescem no âmbito da tecnologia, movimentando anualmente bilhões de dólares em todo o mundo (NOVAK; O'BRIEN; GISH, 2011). Seguindo esse contexto, a primeira palestra realizada no dia 24 de Junho de 2020 , ministrada pelo Patrick Machado, Student Ambassador da Unity, teve como foco as mudanças recorrentes da área dos jogos deste o começo da sua implementação, as atuais e futuras tendências desse ramo. 
Como ilustrado na Figura 1, o palestrante ressaltou os aspectos dos jogos que foram modernizados ao longo do tempo, sendo um dos principais a visualização, desde a evolução do 2D para o 3D, bem como a interatividade que anteriormente era passiva, e que hoje há a realidade aumentada, e também a interação que antecedentemente era apenas com comunicação entre jogador e máquina, tendo nos tempos atuais a possibilidade de multi jogadores.

Figura 1 - Momento da palestra desenvolvimento de jogos

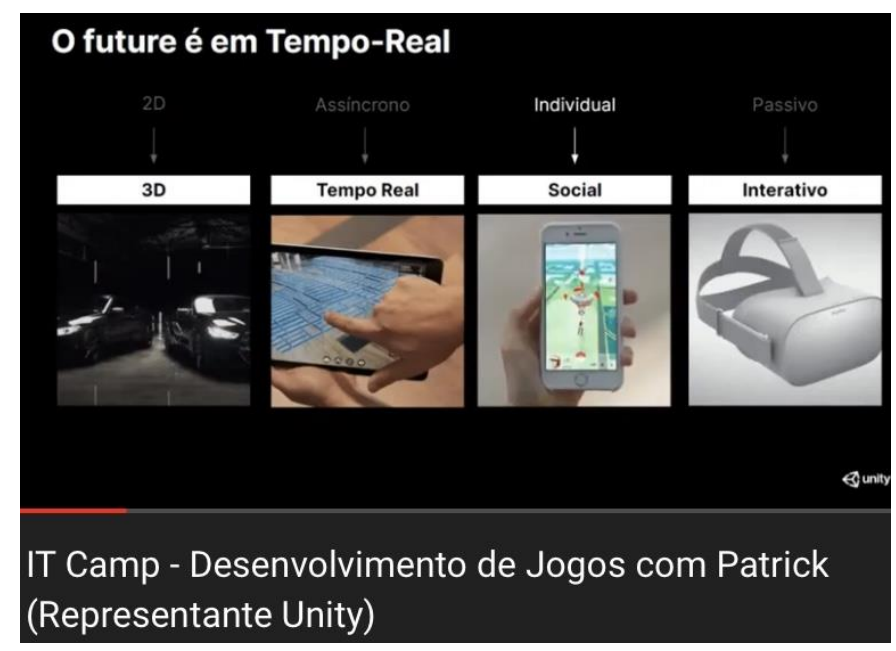

Fonte: Canal do YouTube do Commit Jr.

Nessa prerrogativa, Patrick apresentou as estatísticas do mercado de jogos, as ferramentas mais utilizadas no desenvolvimento de gamers. Não menos importante, explicou os recursos e flexibilidade da plataforma Unity (GOLDSTONE, 2009) na produção de jogos e de materiais audiovisuais. Assim como toda game engine, a Unity, facilita o desenvolvimento de jogos pelo fato do desenvolvedor não precisar programar diretamente para DirectX ou OpenGL.

Para mais, a Unity possui uma grande quantidade de ferramentas, sendo muito fácil de trabalhar com ela, pois além de ser visual, não apenas baseada em código como a Irrlicht, por exemplo, a interface é extremamente amigável. Ela dispõe de uma ferramenta de scripts fundamentada no Mono, ferramenta para desenvolver e executar aplicações.NET em diferentes plataformas, proporcionando a programação em C\#, UnityScript e Boo.

Outro ponto notável da Unity, é a importação de arquivos. Ela importa em muitos formatos, tanto 2D quanto 3D, tirando o trabalho de ter que exportar alguma arte antes de importar na Unity.

\section{Palestra aprendizado de máquina}

"Aprender implica em alterações no sistema que são adaptativas, no sentido que elas capacitam o sistema a realizar a mesma tarefa, ou tarefas provenientes da mesma população, de forma mais eficiente e eficaz na próxima vez" (SIMON, 1983). Nesse sentido, aprendizado de máquina é uma área da Inteligência Artificial (IA) cujo objetivo é o desenvolvimento de técnicas computacionais sobre o aprendizado, bem como a construção de sistemas capazes de adquirir conhecimento de forma automática. Sendo que, um sistema de aprendizado é um programa de computador que toma decisões baseado em experiências acumuladas através da solução bem sucedida de problemas anteriores (MITCHELL; MICHALSKI; CARBONELL, 2013). 
Outrossim, a segunda palestra do IT CAMP, foi ministrada no dia 26 de Junho de 2020, pelo Doutor em Ciência da Computação e docente do CEFET-MG, Daniel Hasan Dalip. O docente introduziu Aprendizado Supervisionado, abordando como compreender o relacionamento entre os atributos e a classe, e em predizer a classe de novos exemplos. Da mesma forma, o Aprendizado Não Supervisionado, ressaltando como encontrar representações úteis dos exemplos, tais quais: agrupamentos (clusters); modelar a densidade dos exemplos; as causas ou as fontes ocultas dos exemplos; redução da dimensão.

Tendo aprendizado de máquina como o "campo de estudo que fornece aos computadores a habilidade de aprenderem sem serem programados de forma explícita" (Samuel, A. L. 1959). O palestrante exemplificou o processo de aprendizagem da máquina, suas metodologias e aplicações de uso. Ademais resolveu uma demonstração prática de um processo de aprendizado de máquina, como ilustra a Figura 2.

Figura 2 - Demonstração prática palestra aprendizado de máquina

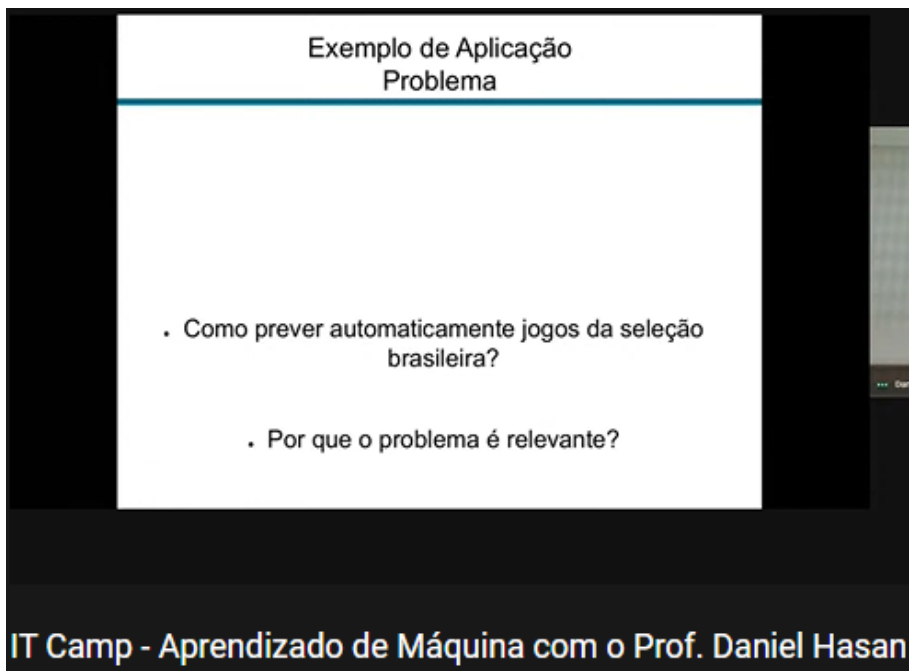

Fonte: Canal do YouTube do Commit Jr.

A apresentação interativa do aprendizado de máquina é considerável porque, quando os modelos são aparente a novos dados, eles são capazes de se adequar independentemente. Aprendem com computações anteriores para produzir soluções e resultados confiáveis, suscetível de repetição. Isso não é considerado uma ciência nova, mas uma ciência que está ganhando um novo estímulo.

\section{Palestra desenvolvimento de back-end}

O termo back-end tem a ideia do que está por trás de uma aplicação, trabalha em boa parte dos casos fazendo a ponte entre os dados que vem do navegador rumo ao banco de dados, sempre aplicando as devidas regras de negócio, validações e garantias em um ambiente onde o usuário final não tenha acesso e possa manipular algo (SMITH, 2012).

A palestra foi ministrada no dia 29 de junho, conduzida pelo Matheus Rosa, Analista da Take, que logo de início levantou a importância do profissional de TI na área, bem como a sua demanda no mercado de trabalho atualmente. Durante a apresentação, Matheus conceituou o desenvolvimento de back-end, discutiu assuntos que rodeiam a área, exibido na Figura 3, como Algoritmos e Estruturas de Dados. 
Figura 3 - Definições da palestra desenvolvimento back-end

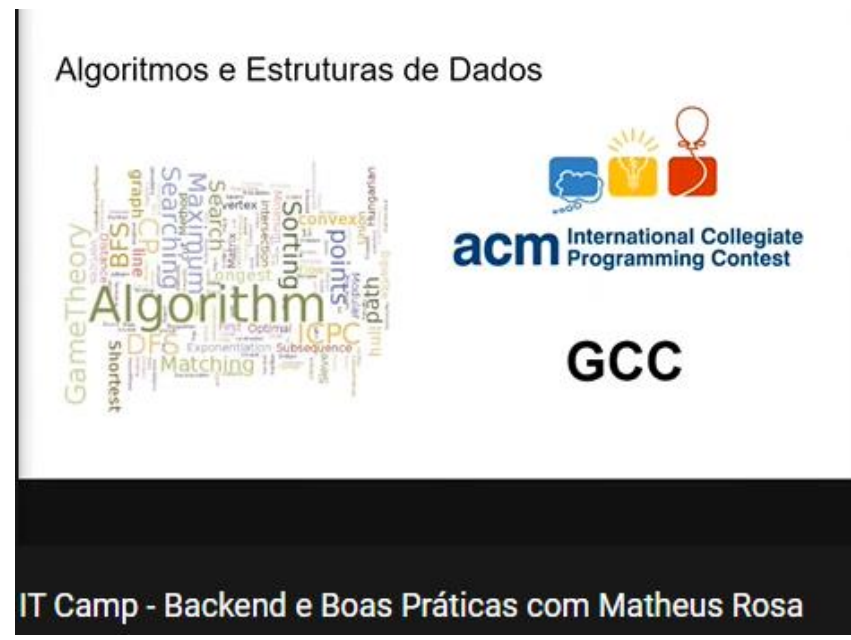

Fonte: Canal do YouTube do Commit Jr.

Para Matheus, o desenvolvimento back-end, é a parte de um sistema computacional ou aplicação que não está diretamente acessada pelo usuário, tipicamente responsável pelo armazenamento e manipulação de dados, em que a lógica de instruções e comandos que faz com que as máquinas façam o que queremos. Por consequência, mesmo sabendo programar utilizando linguagens específicas de programação, é necessário também aprofundar em servidores, banco de dados, APIs, escalabilidade e segurança.

Além disso, detalhou o que seria um bom desenvolvedor de back-end, sendo esse responsável por manter o funcionamento dos websites, coordenar todas as tarefas relacionadas com códigos e linguagens de programação. Tendo como principais funções: fazer o domínio para sistemas operacionais de servidores; analisar informações, relatórios de falhas, dados e estatística dos sites; aplicar técnicas de segurança nos sites; criar bancos de dados e integrá-los com outras aplicações, e não menos importante, saber linguagens específicas de programação, Python, PHP, JavaScript e Ruby.

\section{Palestra criação de site com WordPress e encerramento do IT CAMP}

Adentrando no último dia do IT CAMP, bem como, na palestra de fechamento do evento, houve no dia 01 de julho de 2020 a apresentação das integrantes da CPE Jr., Ana Clara, Laura Costa e Millena Figueiro. Segundo a palestrante, Millena Figueiro, o WordPress é uma das ferramentas mais utilizadas para conteúdo na Web. A popularização deste sistema é devido, entre outros, pelo seu tipo de licença, facilidade de uso, e a versatilidade (WILLIAMS; DAMSTRA; STERN, 2015).

As palestrantes conceituaram o WordPress como sendo um sistema livre e aberto de gestão de conteúdo para a internet, baseado em PHP com banco de dados MySOL, voltado principalmente para a criação de páginas eletrônicas (sites), e blogs online. Sendo, a ferramenta mais utilizada para conteúdo na web, tendo como principal motivo da sua popularização a licença gratuita.

O intuito da palestra, era de introduzir todas as etapas utilizadas na programação de um site utilizando o WordPress. Até mesmo pelo fato dos sites terem se tornado uma necessidade para muitas organizações, negócios e instituições. A princípio deve-se saber a diferença entre o WordPress.com e o WordPress.org, como argumentou a palestrante Laura Costa, o .com é mais utilizado para blogs, sendo pouco flexível, gratuito, mais simples de usar, sem plugins e com serviço de criação e hospedagem de blog. Já o .org é mais 
valido para a criação de sites, sendo mais flexível, 100\% customizável, com milhares de plugins e necessita de certificado SSL, que fornece a segurança do site. Como se encontra representado na Figura 4.

Figura 4 - Palestra sobre criação de site com o WordPress

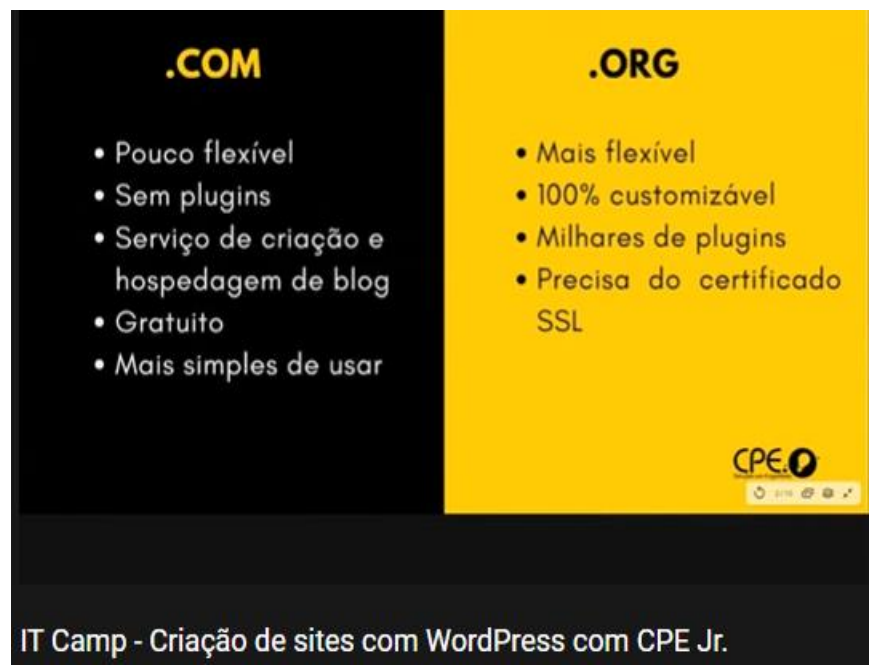

Fonte: Canal do YouTube do Commit Jr.

Mas ainda, foi explicado os pré-requisitos na criação de um site, como possuir um servidor que suporte todos os processos simultâneos; adquirir um nome de domínio, pois irá representar a marca do site como um todo; banco de dados para armazenamento; conexão FTP, que permitirá a troca de arquivos entre dois computadores conectados a internet; e a instalação do WordPress no computador. Da mesma forma, foi abordado a importância dos plugins na construção do site, uma vez que são responsáveis pelos complementos que agregam ou modificam as funcionalidades.

Logo, depois de sanar as dúvidas dos participantes, as palestrantes construíram uma página de um site utilizando o WordPress, ilustrado na Figura 5, a fim de exemplificar na prática como é a elaboração de um website na plataforma.

Figura 5 - Demonstração prática da criação de um site

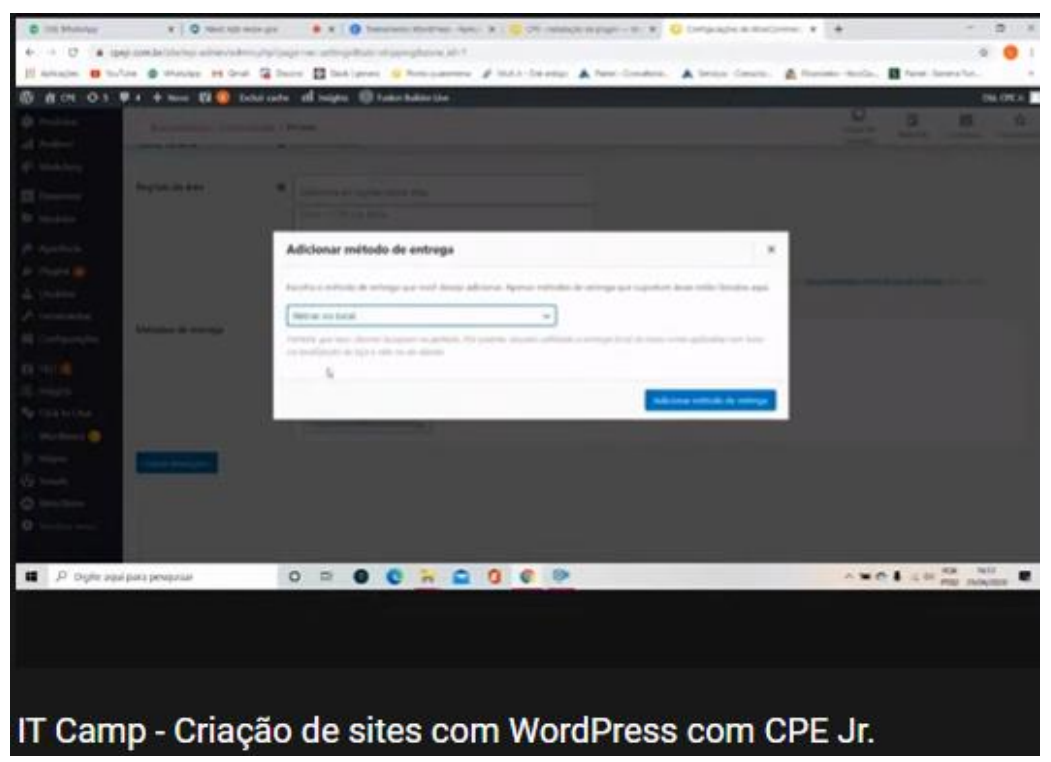

Fonte: Canal do YouTube do Commit Jr. 
Destarte, o IT CAMP teve o seu encerramento com números positivos, com a palestra aprendizado de máquina com mais visualizações, totalizando 363 verificações. A organização do evento, pediu que os presentes respondessem a um formulário de feedback, uma vez que tanto o COMPET quanto o Commit Jr pretendem fazer novas edições do IT CAMP, sendo assim as respostas dos participantes podem contribuir de forma positiva para melhorar e adaptar os eventos futuros. Bem como, foi gerado certificado de participação aos graduandos do CEFET-MG.

\section{CONSIDERAÇÕES FINAIS}

As atividades prescritas nesse trabalho, contribuiu de forma significativa no ensino das 186 pessoas que participaram do IT CAMP, sendo que 117 eram dos cursos de graduação do CEFET-MG, com 103 da Engenharia de Computação; 1 de Engenharia Mecatrônica; 7 da Engenharia de Materiais; 2 da Engenharia Elétrica; 1 da Química tecnológica; 1 da Engenharia Mecânica; 1 de Administração; 1 de Engenharia Ambiental e Sanitária; 8 graduandos de outras instituições; 31 alunos do ensino médio técnico do CEFET-MG e os demais da comunidade externa.

Por consequência, pode-se considerar que o grupo PET da Engenharia de Computação, por meio desse evento, conseguiu atingir os objetivos e metas no que diz respeito ao tríade ensino, pesquisa e extensão, já que a equipe COMPET precisou realizar pesquisas, a fim de auxiliar e contribuir com os palestrantes no conteúdo transferido durante as palestras.

Considerando o ponto de vista dos participantes do IT CAMP, por meio do formulário de avaliação fornecido no encerramento do evento, constatou-se a satisfação pelas notas atribuídas ao evento, sendo que os participantes responderam entre nota 4 (relevante) e 5 (muito relevante), quanto a pergunta "O tema do evento foi relevante para você?".

À luz dessas considerações, conclui-se que, o COMPET vem colaborando significativamente com a comunidade acadêmica e externa, abordando assuntos importantes para o conhecimento profissional dos estudantes, tanto do CEFET-MG, quanto de outras instituições, como também atribuindo conhecimentos práticos e teóricos na formação complementar desses alunos.

\section{Agradecimentos}

Os autores agradecem ao CEFET-MG pelo apoio e fomento dado ao grupo PET, através da Diretoria de Graduação, com a realização da atividade aqui descrita, bem como o auxílio financeiro para a participação e apresentação deste trabalho no COBENGE 2021. Aproveitamos também, para agradecer aos colegas que colaboraram na realização do evento.

\section{REFERÊNCIAS}

CASTELLS, Manuel. A sociedade em rede. São Paulo: Paz e Terra, 1999.

GOLDSTONE, Will. Unity game development essentials. Packt Publishing Ltd, 2009. 
MITCHELL, R.; MICHALSKI, J.; CARBONELL, T. An artificial intelligence approach. Berlin: Springer, 2013.

NOVAK, Jeannie; O'BRIEN, Meaghan; GISH, Jim. Game development essentials. Delmar, 2011.

SAMUEL, A.L. (1959). Some Studies in Machine Learning Using the Game of Checkers. IBM J. Res. Dev., 3, 210-229.

SIMON, H. A., Why Should Machines Learn?, em Machine Learning: An Artificial Intelligence Approach, R. S. Michalski, J. G., and T. M. Mitchell, editores, Tioga, Palo Alto, California, 1983.

SMITH, Peter G. Professional website performance: optimizing the front-end and back-end. John Wiley \& Sons, 2012.

WILLIAMS, Brad; DAMSTRA, David; STERN, Hal. Professional WordPress: design and development. John Wiley \& Sons, 2015.

\section{IT CAMP: Addressing Information Technology}

Abstract: With the objective of serving the academic and external community, the PET group, Tutorial Education Program, of Computer Engineering (COMPET) of CEFET - MG located at the Nova Gameleira Campus in Belo Horizonte, includes introducing themes that involve information technologies, promoting an event, which, through lectures, sought to highlight issues that involve the labor market, as well as to update participants on new technologies. The event was named IT CAMP, with lectures on game development; creation of wordpress website; back-end development and machine learning. Thus, COMPET finds a partnership with Commit Jr, a junior company in the Work Engineering course at CEFETMG, to carry out the activities of the event that took place between June 24th and July 1st, 2020, on the youtube jr commit channel.

Keywords: PET. Computer engineering. Teaching. Extension. Junior company. 